\title{
Hérnia discal cervical não traumática e Síndrome de Brown-Séquard. Relato de caso e revisão da literatura
}

\author{
Cléciton Braga Tavares ${ }^{1}$, Emerson Brandão Sousa', Igor Brenno Campbell Borges ${ }^{1}$, \\ Baldomero Pinto Soares ${ }^{2}$, Nelson Geraldo Freire Neto ${ }^{3}$
}

Unidade de Neurocirurgia do Hospital de Base do Distrito Federal, Brasília, DF, Brasil e Universidade Estadual do Piauí, Teresina, PI, Brasil.

\section{RESUMO}

A Síndrome de Brown-Séquard (SBS) é caracterizada pela perda da função motora, propriocepção e sensibilidade vibratória ipsilateral e perda da sensibilidade tátil e dolorosa contralateral à hemissecção medular. É principalmente causada por fraturas da coluna vertebral ou tumores extramedulares. Hérnia discal cervical não traumática é uma etiologia rara, havendo 31 relatos em literatura indexada até o momento. Paciente do sexo masculino, 23 anos, admitido com parestesia em dimídio esquerdo e fraqueza no hemicorpo direito há cerca de 35 dias da internação. Sem relatos de trauma. Ao exame: consciente e orientado, hemiparesia à direita e hemi-hipoestesia tátil dolorosa à esquerda com nível motor e sensitivo em C7. Os exames de imagem evidenciaram um canal estreito cervical de C4-T1, presença de hérnia discal extrusa C5-C6 e hipersinal medular a esse nível. Foi submetido à discectomia e artrodese cervical anterior de C5-C6. No pós-operatório, evoluiu com tetraplegia flácida (nível motor/sensitivo em C8). Os exames de controle mostraram correto posicionamento do instrumental cirúrgico, ausência de hérnias discais e manutenção do hipersinal medular. Após oito meses de reabilitação e seguimento ambulatorial, permanece tetraparético. Descrevemos o primeiro caso brasileiro, em literatura indexada, de SBS causada por hérnia discal cervical não traumática. Há um predomínio pelo sexo masculino, a média de idade é de 45 anos e o disco intervertebral C5-C6 é o mais acometido. Microdiscectomia e fusão intersomática são as formas mais comuns de tratamento. Após a descompressão precoce, há um bom prognóstico, com recuperação da motricidade na maioria dos casos.

\section{PALAVRAS-CHAVE}

Síndrome de Brown-Séquard, deslocamento do disco intervertebral, discotomia, traumatismos da coluna vertebral.

\section{ABSTRACT}

Nontraumatic cervical discal herniation and Brown-Séquard's Syndrome. Case report and literature review

The Brown-Séquard's Syndrome is characterized by loss of motor function, proprioception and vibration sensitivity ipsilateral and loss of tactile and painful contralateral to hemisection spinal cord. It is mainly caused by fractures of the spine or extramedullary tumors. Nontraumatic cervical herniated disc etiology is rare, with only 31 cases indexed in the literature. Male patient, 23 years old, admitted with numbness in left side and weakness in the right hemisphere, hospitalized for about 35 days. No reports of trauma. On examination: conscious and oriented, right hemiparesis and hemihipoestesia tactile-painful in left side, with sensory and motor level C7. Imaging tests showed a narrow channel of cervical C4-T1, presence of disc herniation extrusa C5-C6 and hyperintense marrow at this level. Underwent cervical discectomy and arthrodesis of C5-C6. Postoperatively evolved with flaccid tetraplegia (level motor/sensory C8). The control examinations showed correct position surgical instruments, lack of disc herniations and maintenance of spinal cord hyperintense. After eight months of rehabilitation and outpatient services remains tetraparesis. To the authors' best knowledge, this is the first description of BSS caused by nontraumatic cervical disc herniation in Brazil, in the indexed literature. In the affected patients, there is a predominance of males, with mean age 45 years, and the C5-C6 intervertebral disc is the most affected. Microdiscectomy and interbody fusion are the most common forms of treatment. After early decompression, there is a good prognosis, with recovery of motor function in most cases.

\section{KEYWORDS}

Brown-Séquard Syndrome, intervertebral disc displacement, diskectomy, spinal injuries.

1 Médico neurocirurgião e professor da Disciplina de Neurologia/Neurocirurgia da Universidade Estadual do Piauí, Teresina, PI, Brasil.

2 Médico-residente de Neurocirurgia do Hospital de Base do Distrito Federal, Brasília, DF, Brasil.

3 Médico neurocirurgião e preceptor do Programa de Residência Médica em Neurocirurgia do Hospital de Base do Distrito Federal, Brasília, DF, Brasil. 


\section{Introdução}

A Síndrome de Brown-Séquard (SBS) ou hemissecção medular é caracterizada pela perda da função motora, propriocepção e sensibilidade vibratória ipsilateral (devido a lesão do trato corticoespinhal e fascículo grácil e cuneiforme) e perda da sensibilidade tátil e dolorosa contralateral (resultado da lesão dos tratos espinotalâmicos). ${ }^{1-3}$

É principalmente causada por fraturas da coluna vertebral ou tumores extramedulares que comprimem e lesam a medula espinhal. Hérnia discal cervical não traumática é uma etiologia rara, havendo 31 relatos em literatura indexada até o momento. Os três primeiros casos foram descritos em 1928, por Stookey. ${ }^{1,3-5}$

Apresentamos o primeiro relato brasileiro em literatura indexada de Síndrome de Brown-Séquard causada por hérnia discal cervical não traumática. Essa descrição também tem a importância de nos lembrar que patologias degenerativas da coluna vertebral podem se manifestar das mais variadas formas e acometer as diversas faixas etárias.

\section{Relato do caso}

Paciente do sexo masculino, 23 anos, admitido no Serviço de Emergência da Unidade de Neurocirurgia do Hospital de Base do Distrito Federal (Brasília-DF) com relato de parestesia em dimídio esquerdo e fraqueza no hemicorpo direito há cerca de 35 dias da internação. Sem episódios prévios de trauma.

Ao exame de admissão: consciente e orientado, pupilas isocóricas e fotorreagentes, hemiparesia à direita e hemi-hipoestesia tátil dolorosa à esquerda com nível motor e sensitivo em C7.

Os exames de imagem evidenciaram um canal estreito cervical de $\mathrm{C} 3-\mathrm{C} 7$ e a presença de hérnia discal extrusa C5-C6 e hipersinal medular a esse nível (Figuras 1 e 2).

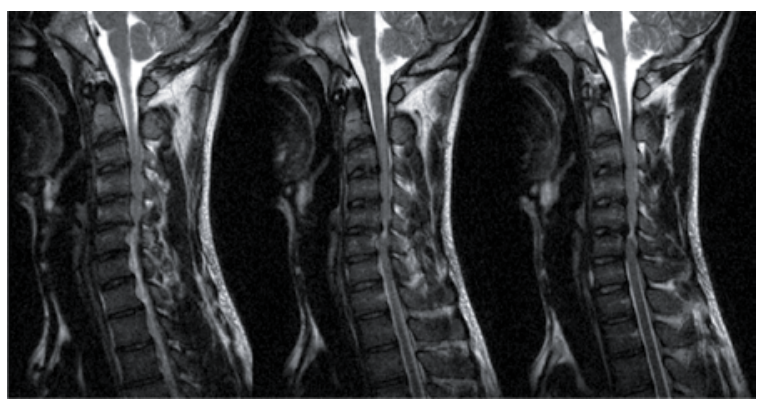

Figura 1 - Ressonância magnética de coluna cervical em cortes sagitais mostrando canal estreito cervical entre C3-C7 e hérnia discal C5-C6 com hipersinal medular.

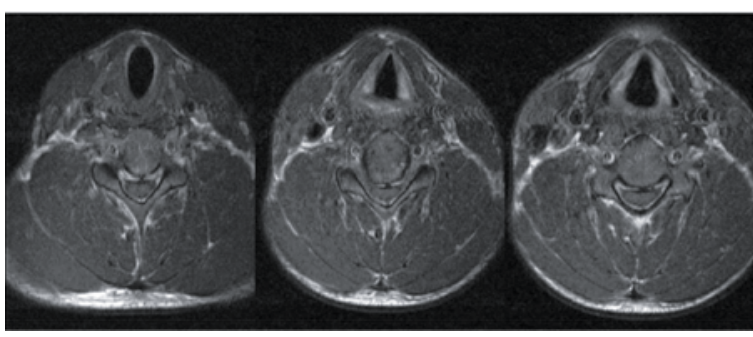

Figura 2 - Ressonância magnética de coluna cervical em cortes axiais mostrando hérnia discal C5-C6, com diminuição importante do canal raquidiano.

Foi submetido a discectomia e artrodese cervical anterior de C5-C6, com interposição de enxerto ósseo autólogo de crista ilíaca e placa com 4 parafusos.

No pós-operatório evoluiu com tetraplegia flácida (nível motor/sensitivo em C8), infecção urinária e pneumonia. Fez uso de antibioticoterapia de largo espectro por 10 dias e fisioterapia motora/respiratória. Os exames de imagem de controle mostraram correto posicionamento do instrumental cirúrgico, ausência de hérnias discais e manutenção do hipersinal medular.

Após oito meses de reabilitação e seguimento ambulatorial, permanece tetraplégico com nível motor e sensitivo em C8.

\section{Discussão}

Descrevemos o primeiro caso brasileiro de SBS causada por hérnia discal cervical extradural não traumática. Neste relato, o paciente não apresenta um déficit motor e sensitivo completo, até porque a hemissecção incompleta é a forma mais comum de apresentação. ${ }^{1}$

Há um predomínio pelo sexo masculino, a média de idade é de 45 anos, variando entre 25 e 73 anos, e o disco intervertebral C5-C6 é o mais acometido. As hérnias discais extradurais são bem mais frequentes. As intradurais representam cerca de $0,3 \%$ de todas as hérnias discais e apenas 3\% das herniações cervicais. ${ }^{1}$ Faixa etária, nível vertebral e topografia raquidiana condizentes com este relato.

Observamos em nosso caso que o nível sensitivo encontrava-se abaixo do nível vertebral da lesão. Essa discordância tem sido presenciada em outros trabalhos. Isso pode ser explicado pelo fato de o trato espinotalâmico lateral cruzar a medula espinhal dois ou três segmentos acima do seu nível de entrada. Adicionalmente, pode estar relacionado à severidade da compressão medular e das variações anatômicas individuais da artéria espinhal anterior. ${ }^{1}$

Microdiscectomia e fusão intersomática são as formas mais comuns de tratamento, principalmente 
nas hérnias intradurais. ${ }^{1,2}$ Em nosso paciente, realizamos discectomia e artrodese cervical anterior com interposição de enxerto ósseo autólogo e placa com parafusos. Entre as indicações cirúrgicas adotadas e outros trabalhos, destacam-se laminectomia, hemilaminectomia, microdiscectomia sem fusão intersomática, corpectomia e fusão intersomática, discectomia com laminectomia e foraminotomia anterior. ${ }^{1-4,6-10}$

Após o tratamento cirúrgico precoce acrescido à fisioterapia, há um bom prognóstico, com recuperação da motricidade na maioria dos casos. Poucos pacientes mantêm um déficit neurológico residual ${ }^{1-4,6-8}$, fato não ocorrido neste caso. O paciente evoluiu com piora após a descompressão e o déficit mantém-se após dois meses de seguimento ambulatorial, provavelmente em decorrência da demora na realização da descompressão cirúrgica.

\section{Conflitos de interesse}

Os autores declaram não haver conflitos de interesse.

\section{Referências}

1. Lee JK, Kim YS, Kim SH. Brown-Sequard syndrome produced by cervical disc herniation with complete neurologic recovery: report of three cases and review of the literature. Spinal Cord. 2007;45(11):744-8.
2. Clatterbuck RE, Belzberg AJ, Ducker TB. Intradural cervical disc herniation and Brown-Séquard's syndrome. Report of three cases and review of the literature. J Neurosurg. 2000;92(Suppl 2):236-40.

3. Sayer FT, Vitali AM, Low HL, Paquette S, Honey CR. Brown-Séquard syndrome produced by $\mathrm{C} 3-\mathrm{C} 4$ cervical disc herniation: a case report and review of the literature. Spine (Phila Pa 1976). 2008;33(9):E279-82.

4. Mastronardi L, Ruggeri A. Cervical disc herniation producing Brown-Séquard syndrome: case report. Spine (Phila Pa 1976). 2004;29(2):E28-31.

5. Lim E, Wong YS, Lo YL, Lim SH. Traumatic atypical BrownSequard syndrome: case report and literature review. Clin Neurol Neurosurg. 2003;105(2):143-5.

6. Börm W, Bohnstedt T. Intradural cervical disc herniation. Case report and review of the literature. J Neurosurg. 2000;92(Suppl 2):221-4.

7. Mustapha AF, Aremu AA, Bello TO. Cervical disc herniation and Brown-Sequard syndrome: a case report. Afr J Med Med Sci. 2008;37(2):193-7.

8. Kobayashi N, Asamoto S, Doi H, Sugiyama H. BrownSéquard syndrome produced by cervical disc herniation: report of two cases and review of the literature. Spine J. 2003;3(6):530-3.

9. Antich PA, Sanjuan AC, Girvent FM, Simó JD. High cervical disc herniation and Brown-Sequard syndrome. A case report and review of the literature. J Bone Joint Surg Br. 1999;81(3):462-3.

10. Rumana CS, Baskin DS. Brown-Séquard syndrome produced by cervical disc herniation: case report and literature review. Surg Neurol. 1996;45(4):359-61.

\section{Endereço para correspondência}

Cléciton Braga Tavares

SQS 303, Bloco A, ap. 206, Asa Sul

70336-010 - Brasília, DF, Brasília

E-mail: bragatavares@yahoo.com.br 